\title{
Circulating Soluble-Klotho, Exercise, and the Cardiovascular System
}

\author{
Saghiv $\mathbf{M}^{*}$ \\ Department of Human Performance and Leisure Studies, USA
}

*Corresponding author: Saghiv M, Associate Professor of Clinical Exercise Physiology, Chair, Department of Human Performance and Leisure Studies, College of Health and Human Sciences, NC A\&T State University, USA.

Keywords: Exercise modalities; Soluble klotho; Cardiovascular system

\section{Introduction}

It has long been established that anaerobic and aerobic exercise both acutely and chronically influence the cardiovascular system, its capacity for work, efficiency, and more [1]. Furthermore, exercise has been identified as one of multiple means to decrease the occurrence of aging, and limit the symptoms assigned with the ageing syndrome [2].

\section{The Ageing Syndrome}

Often, aging (or ageing) is regarded as a syndrome. Aging has been addressed as a syndrome presenting with a group of clinical and non-clinical symptoms and processes that decrease or increase as one's age advances [3]. Olson CB suggested that aging is a multifactorial process [4] while de Magalhães JP (2004) suggested that aging originates and occurs at the cellular level [5].

\section{S-Klotho}

The Klotho gene was discovered in rats in the year 1997 by Kuro-o et al. [6]. Klotho has been identified a bit more than two decades ago as an ageing influencer (for better or for worse) $[6,7]$. While plenty of data exist regarding Klotho and S-Klotho in general, relatively few data exist regarding the mutual effects of exercise on S-Klotho and S-Klotho on exercisability. The Klotho protein has two forms: one as a membrane protein, and the other in secreted form [8]. The researchers reported the gene had several ageing suppressing properties. The researchers indicated that the gene encodes for a membrane protein with similarities in its sequence to those of enzymes from the beta-glucosidase family [6]. The protein was suggested as part of a signaling pathway that is influential in age-related diseases' morbidity and the regulation of ageing in vivo [6]. Kuro-o et al. further indicated a defect in the gene may lead to a shorter lifespan, infertility, Arteriosclerosis, skin atrophy, Osteoporosis, Emphysema, and a syndrome resembling that of human ageing [6]. Age-related declines are manifested by a decreased ability for aged skeletal muscle to respond to physiological stimuli such as muscle loading or acute injury, and disease related effects $[9,10]$. Klotho gene expression in positively influenced by different exercise modalities acting as an epigenetic influencer [11], resulting in increased work capacity, performance, and lifespan, and decreased aging in healthy and diseased populations [12-14]. As is the case with exercise, positive adaptations decrease and diminish with time if exercise training is not maintained.

\section{Exercise Modalities and S-Klotho}

Regular aerobic exercise participation promotes health and disease prevention [15]. Endurance exercise such as biking, walking, swimming and running, appear to benefit longer life expectancy than anaerobic exercise such as power lifting [16]. It has been suggested that circulating S-Klotho levels are upregulated in response to an acute exercise bouts, but that the response may be dependent on a person's fitness level [17-20]. Compared to sedentary young and old subjects, in the elite well aerobic trained young runners and master athletes S-Klotho levels are markedly elevated while [21] IGF-I is generally thought to be associated with anabolism and wellbeing [22], yet, signaling through IGF-I and Insulin receptors is negatively related to adults [23]. 


\section{S-Klotho and the Cardiovascular System}

Several clinical studies have suggested that the Klotho gene exerts strong cardio-protective effects. S-Klotho has been proposed as a key regulator of the development of cardiovascular disease. An association between low levels of S-Klotho and the occurrence and severity of cardiovascular disease have been reported, as well as a reduction of cardiovascular risk when levels were high [24]. This protein is related to the attenuation of vascular calcification as well as prevention of cardiac hypertrophy.

Semba RD et al. [25] concluded that in community-dwelling adults, higher plasma klotho concentrations are independently associated with a lower likelihood of having cardiovascular disease [25].

Nagai R \& Hoshino Y [26] reviewed the new knowledge (at the time of publishing their review) in regard to the genetics of cardiovascular disease. In their article they wrote "We recently found a novel gene which seems to affect human aging phenotype and vascular endothelial function. It is important as a future study to clarify the regulatory mechanisms of the klotho gene in the cardiovascular system and the clinical significance of klotho gene polymorphisms" [26].

Saito Y et al. [27] reported regarding Klotho's influence of human vascular endothelial tissue, suggesting it has a protective role. The researchers indicated the secreted form of Klotho is of cDNA, and the lack of knowledge as to its pathophysiological relevancy. In their conclusion the authors wrote "These results suggest that the Klotho protein protects the cardiovascular system through endotheliumderived NO production by humoral pathways" [27].

Skrzypkowska M et al. [28] suggested a possible mechanism explaining Klotho KL-VS polymorphism's influence on ageing and cardiovascular disease development. These researchers suggested that individuals possessing at least one KL-VS allele are characterized by greater number of CD34+ and CD34+VEGFR2+ and their various subpopulations (CD34+CD133+, CD34+c-Kit+, CD34+CXCR4+ and CD34+VEGFR2+c-Kit+) than wild-type". The researchers came to the conclusion that "One of the mechanisms that are responsible for previously described KL-VS heterozygote advantage may be connected with maintaining greater size of hematopoietic and endothelial progenitor cells population" [28].

Serum klotho was an independent biomarker of LVMI but not arterial stiffness and vascular calcification. Further studies are warranted to elucidate the clinico-pathogenic significance of klotho for cardiovascular parameters, and whether any interventions to maintain or increase the serum klotho level can prevent cardiovascular events and mortality in CKD patients [29].

\section{S-Klotho, Exercise, and the cardiovascular System}

The research of the relationship between exercise modalities and S-klotho in health and disease is on the rise. The combination of two research findings whereas S-Klotho had cardiovascular protective characteristics and that exercise modalities have an influence on S-Klotho levels, leads one to the logical conclusion that exercise will influence for the better one's cardiovascular clinical condition via increase of S-Klotho.
Saghiv et al. [30]concluded that "Inflection of Klotho expression through aerobic exercise training represents an interesting relationship that may contribute to the explanation of the antiaging and anti-CAD effects of long-lasting aerobic activity. Both are factors that may promote upgrading capacities of the elderly, CAD patients and healthy young adult subjects. Accordingly, a longlasting aerobically trained individual is associated with decreased risk factors and increased s-Klotho that clearly counteracts the action of IGF-I. Following anaerobic exercise training, there is no association with circulating s-Klotho; however, it is a potent stimulus to increase plasma IGF-I levels".

In their conclusions Matsubara T et al. [31] wrote "a correlation between plasma Klotho concentration and arterial stiffness, as well as aerobic exercise capacity. Furthermore, aerobic exercise training increased plasma Klotho concentration and decreased arterial stiffness. The decrease in the arterial stiffness was associated with an increase in plasma Klotho concentration. These results suggest that aerobic exercise training induced an increase in plasma Klotho concentration, which in turn, could contribute to a decrease in arterial stiffness".

\section{Conclusion}

Both exercising, especially aerobically, and high levels of $\mathrm{S}$-Klotho, decrease the chances of the occurrence of cardiovascular disease. Exercise increases the levels of S-Klotho, which in turn, decreases evermore the chances of cardiovascular disease.

\section{Acknowledgement}

None.

\section{Conflict of Interest}

No conflict of interest.

\section{References}

1. Shashi K Agarwal (2012) Cardiovascular benefits of exercise. Int J Gen Med 5: 541-545.

2. Garatachea N, Pareja Galeano H, Sanchis-Gomar F, Santos Lozano A Fiuza Luces C, et al. (2015) Exercise Attenuates the Major Hallmarks of Aging. Rejuvenation Res 18(1): 57-89.

3. Marengoni A, Angleman S, Melis R, Mangialasche F, Karp A, et al. (2011) Aging with multimorbidity: a systematic review of the literature. Ageing Res Rev 10(4): 430-439.

4. Olson CB (1987) A review of why and how we age: a defense of multifactorial aging. Mech Ageing Dev 41(1-2): 01-28.

5. de Magalhães JP (2004) From cells to ageing: a review of models and mechanisms of cellular senescence and their impact on human ageing. Exp Cell Res 300(1): 01-10.

6. Kuro-o M, Matsumura Y, Aizawa H, Kawaguchi H, Suga T, et al. (1997) Mutation of the mouse klotho gene leads to a syndrome resembling ageing. Nature 390(6655): 45-51.

7. Matsumura Y, Aizawa H, Shiraki-Iida T, Nagai R, Kuro-o M, et al. (1998) Identification of the human klotho gene and its two transcripts encoding membrane and secreted klotho protein. Biochemical and Biophysical Research Communications 242(3): 626-630.

8. Kuro 0 (2011) Klotho and the Aging Process. Korean J Intern Med 26 113-122.

9. Wang Y, Sun Z (2009) Current Understanding of Klotho. Ageing Res Rev 8: 43-51.

10. Wang Y, Sun Z (2014) Antiaging Gene Klotho Regulates Endothelin-1 Levels and Endothelin Receptor Subtype B Expression in Kidneys of 
Spontaneously Hypertensive Rats. Journal of hypertension 32: 1629 1636.

11. Ling C, Ronn T (2014) Epigenetic Adaptation to Regular Exercise in Humans. Drug Discov Today 19: 1015-1018.

12. Denham J, Marques FZ, O’Brien BJ, Charchar FJ (2014) Exercise: Putting Action into Our Epigenome. Sports Med 44: 189-209.

13. Ao Bian, Javier A Neyra, Ming Zhan, Ming Chang Hu (2015) Klotho, Stem Cells, and Aging. Clin Interv Aging 10: 1233-1243.

14. Matsubara T, Miyaki A, Akazawa N, Choi Y, Ra SG, et al. (2014) Aerobic Exercise Training Increases Plasma Klotho Levels and Reduces Arterial Stiffness in Postmenopausal Women. Am J Physiol Heart Circ Physiol 306: H348-H355.

15. Paffenbarger RS, Blair SN, Lee IM (2001) A History of Physical Activity, Cardiovascular Health and Longevity: The Scientific Contributions of Jeremy N Morris, DSc, DPH, FRCP. Int J Epidemiol 30(5): 1184-92.

16. Schefer V, Talan MI (1996) Oxygen Consumption in Adult and AGED C57BL/6J Mice during Acute Treadmill Exercise of Different Intensity. Exp. Gerontol 31: 387-392.

17. Phelps M, Pettan-Brewer C, Ladiges W, Yablonka-Reuveni Z (2013) Decline in Muscle Strength and Running Endurance in Klotho Deficient C57BL/6 Mice. Biogerontology 14: 729-739.

18. Saghiv M (2015) Effects of Aerobic Exercise Training on S-Klotho in Young and Elderly. J J Physiology 1(1): 001.

19. Lee EY, Kim SS, Lee JS, Kim IJ, Song SH, et al. (2014) Soluble $\alpha$-Klotho as a Novel Biomarker in the Early Stage of Nephropathy in Patients with Type 2 Diabetes. Plos One 9(8): e102984.

20. Bonnefoy M, Patricot MC, Lacour JR, Rahmani A, Berthouze S, et al (2002) Relation between Physical Activity, Muscle Function and IGF-I, Testosterone and DHEAS Concentrations in the Elderly. [Article in French] Rev Med Interne 23: 819-827.

21. Berryman DE, Christiansen JS, Johannsson G, Thorner MO, Kopchick JJ, et al. (2008) Role of the GH/IGF-I Axis in Lifespan and Health Span: Lessons from Animal Models. Growth Horm IGF Res 18: 455-471.
22. Ahima RS, Prabakaran D, Mantzoros C, Qu D, Lowell B, et al. (1996) Role of Leptin in the Neuroendocrine Response to Fasting. Nature 382: 250252.

23. Château MT, Araiz C, Descamps S, Galas S (2010) Klotho Interferes with a Novel FGF-Signaling Pathway and Insulin/IGF-Like Signaling to Improve Longevity and Stress Resistance in Caenorhabditis Elegans. Aging 2: 567-581.

24. Martín-Núñez E, Donate-Correa J, Muros-de-Fuentes M, Mora-Fernández C, Navarro-González JF, et al. (2014) Implications of Klotho in Vascular Health and Disease. World J Cardiol 6: 1262-1269.

25. Semba RD, Cappola AR, Sun K, Bandinelli S, Dalal M, et al. (2011) Plasma Klotho and Cardiovascular Disease in Adults. J Am Geriatr Soc 59(9): 1596-1601.

26. Nagai R, Hoshino Y (1998) Molecular genetics of cardiovascular diseases. Rinsho Byori 46(3): 249-257.

27. Saito Y, Kuroo M, Nabeshima Y, Nagai R (1999) The protective role of Klotho gene on vascular endothelium. Nihon Rinsho 57(7): 1514-1518.

28. Skrzypkowska M, Słomiński B, Ryba-Stanisławowska M, Gutknecht P, Siebert J, et al. (2018) Circulating CD34+ and CD34+VEGFR2+ progenitor cells are associated with KLOTHO KL-VS polymorphism. Microvasc Res S0026-2862(17): 30212-1

29. Kim HJ, Kang E, Oh YK, Kim YH, Han SH, et al. (2018) The association between soluble klotho and cardiovascular parameters in chronic kidney disease: results from the KNOW-CKD study. BMC Nephrol 19: 51

30. Saghiv MS, Sira DB, Goldhammer E, Sagiv M (2017) The effects of aerobic and anaerobic exercises on circulating soluble-Klotho and IGF-I in young and elderly adults and in CAD patients. J Circ Biomark 6: 1849454417733388.

31. Matsubara T, Miyaki A, Akazawa N, Choi Y, Ra SG, et al. (2014) Aerobic exercise training increases plasma Klotho levels and reduces arterial stiffness in postmenopausal women. Am J Physiol Heart Circ Physiol 306: H348-H355. 